\title{
Physicochemical Characterization of Oleanolic Acid- Human Serum Albumin Complexes for Pharmaceutical and Biosensing Applications
}

Katarzyna Dopierała $^{1 *}$, Martyna Krajewska ${ }^{1}$, Marek Weiss ${ }^{2}$

1- Institute of Chemical Technology and Engineering, Poznan University of Technology, Berdychowo 4, 60-965 Poznań, Poland

2- Institute of Physics, Poznan University of Technology, Piotrowo 3, 60-965 Poznań, Poland *corresponding author, E-mail: katarzyna.dopierala@put.poznan.pl

\section{Supplementary material including 2 pages, 3 figures.}

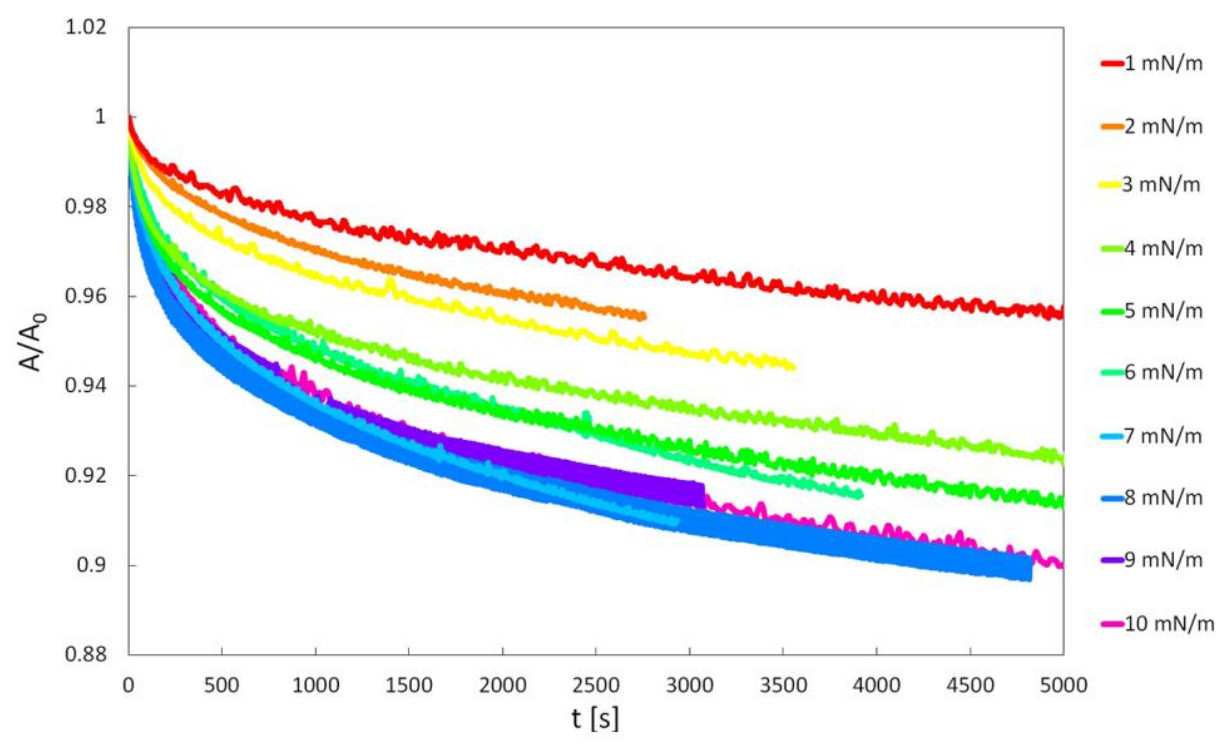

Figure SM1 The relaxation curves for oleanolic acid monolayer compressed to various surface pressures. 

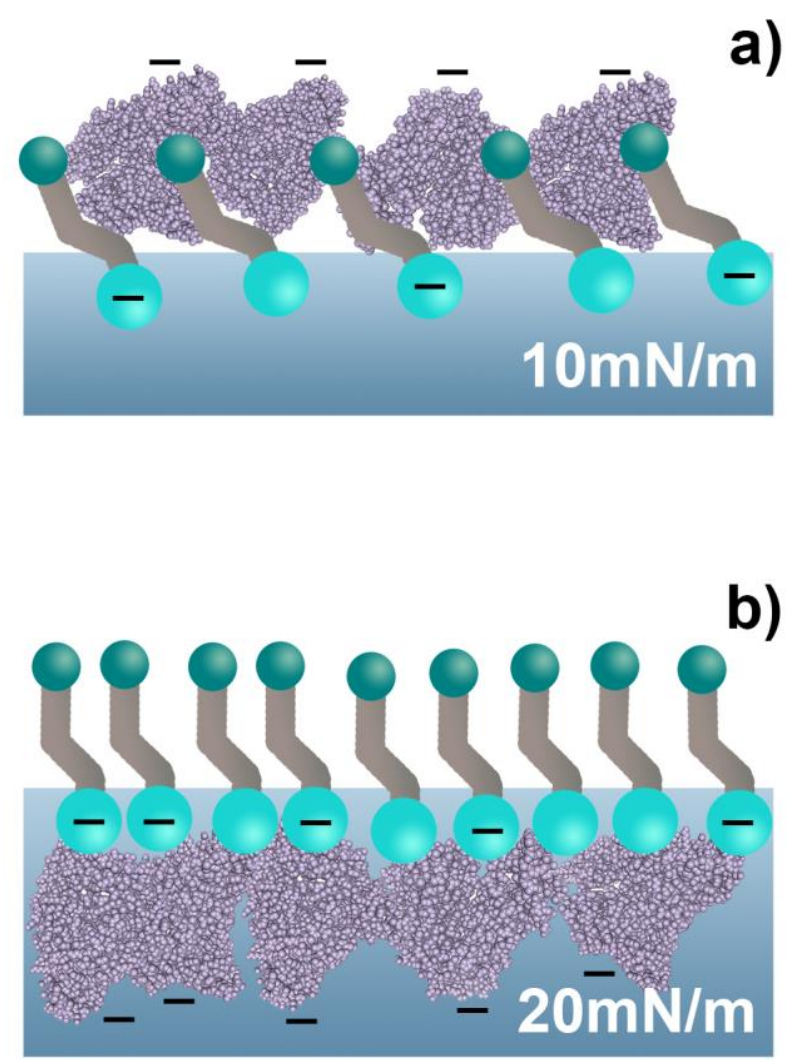

Figure SM 2 Schematic representation of bilayer structure of OLA+HSA film formed in expanded a) and condensed state b).

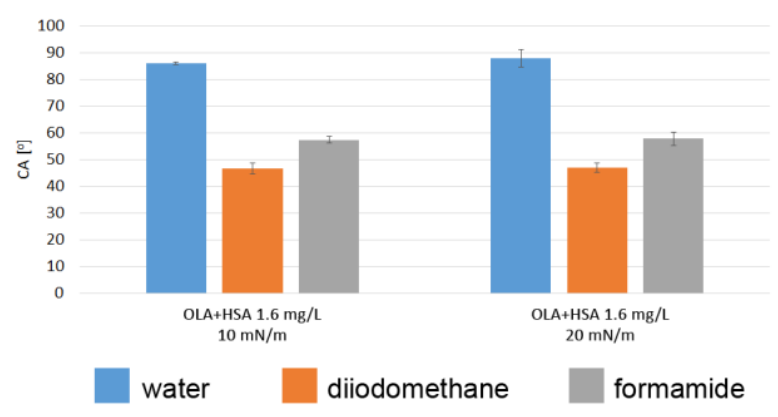

Figure SM 3 Water, diiodomethane and formamide contact angle values for the LB/LS films of OLA+HSA transferred at different surface pressures. 